\title{
Kaposi sarcoma associated herpesvirus infection of primary human endothelial cells activates the proto-oncogene STAT3
}

\author{
Christine A King $^{1 *}$, Craig McCormick ${ }^{2}$ \\ From 13th International Conference on Malignancies in AIDS and Other Acquired Immunodeficiencies \\ (ICMAOI) \\ Bethesda, MD, USA. 7-8 November 2011
}

Kaposi's sarcoma associated herpesvirus (KSHV) in the etiological agent for 3 AIDS-related cancers: Kaposi's sarcoma (KS), primary effusion lymphoma, and multicentric Castleman's disease. The molecular mechanisms used by KSHV to induce cancer are incompletely understood. KS lesions harbor proliferating latently-infected endothelial cells (ECs), large numbers of inflammatory cells, and marked neoangiogenesis. Considered the major driving force in the development of KS, these KSHV-infected ECs elaborate a variety of pro-inflammatory and angiogenic factors that contribute to tumourigenesis. Considerable evidence has accumulated suggesting a critical role for activated signal transducer and activator of transcription-3 (STAT3) in malignant transformation. STAT3 is a latent transcription factor that upon activation, drives the expression of a number of genes involved in cell proliferation, survival, and immune responses. Canonical STAT3 activation occurs via phosphorylation of Y705, dimerization, and nuclear translocation, followed by phosphorylation of $\mathrm{S} 727$ for maximal transcriptional activity. Activated STAT3 has been observed in a variety of malignancies and has been shown to induce fibroblast transformation in vitro suggesting that STAT3 is a protooncogene. Interestingly, evidence has accumulated suggesting a role for S727 mono-phosphorylated STAT3. Here we show that latent KSHV infection of primary human endothelial cells (ECs) in vitro activates STAT3, and identify a key latency protein, kaposin B, that contributes to this activation. Kaposin B expression in ECs causes STAT3 phosphorylation at S727, in the absence of significant Y705 phosphorylation, and enhanced expression of a subset of STAT3 target genes including CCL5. Recent work shows that the tripartite motif-containing protein 28 (TRIM28, a.k.a. TIF-1 $\beta$, KAP-1) negatively regulates STAT3 by recruiting transcriptional silencing complexes. The repressive activity of TRIM28 is mediated by post-translational modifications and a key site in the regulation of repressor activity maps to S473. Phosphorylation of this residue disrupts the recruitment of transcriptional silencing complexes effectively deactivating the co-repressive function of TRIM28. Confocal microscopy and western blot analysis demonstrate phosphorylation of TRIM28 at S473 in KSHV latently infected and kaposin B expressing ECs. Taken together, our studies suggest kaposin B may contribute to tumourigenesis via constitutive activation of STAT3.

\section{Author details}

'Department of Microbiology and Immunology, SUNY Upstate Medical University, Syracuse, NY, USA. ${ }^{2}$ Department of Microbiology and Immunology, Dalhousie University, Halifax, NS, Canada.

Published: 19 April 2012

\section{doi:10.1186/1750-9378-7-S1-P38}

Cite this article as: King and McCormick: Kaposi sarcoma associated herpesvirus infection of primary human endothelial cells activates the proto-oncogene STAT3. Infectious Agents and Cancer 2012 7(Suppl 1):P38.

\footnotetext{
* Correspondence: kingch@upstate.edu

'Department of Microbiology and Immunology, SUNY Upstate Medical

University, Syracuse, NY, USA

Full list of author information is available at the end of the article
}

( 2012 King and McCormick; licensee BioMed Central Ltd. This is an Open Access article distributed under the terms of the Creative 